\title{
ADDITIONAL GRAVITATIONAL FORCE IS ACTING ON SOME TREES DUE TO PRESENCE OF UNDERGROUND WATER STREAM
}

\author{
MORE B.M.
}

Brahmdevdada Mane Institute of Technology, Belati, Solapur- 413002, MS, India.

${ }^{*}$ Corresponding Author: Email- babasahebmore@gmail.com

Received: May 10, 2012; Accepted: June 04, 2012

\begin{abstract}
An unusual growth is developed with some trees. A bunch of some of their twigs is bending in the downward direction and twigs are stretched downward. Bending of twigs is due to additional gravitational force acting on these twigs. This additional gravitational force is because of presence of underground water stream. The direction of plane in which the twigs are bending indicates the ground surface line where underground water stream is present.
\end{abstract}

Key words- Unusual growth, Twigs of tree, Gravitational Force, Underground Water stream.

Citation: More B.M. (2012) Additional Gravitational Force is Acting on some Trees due to Presence of Underground Water Stream. International Journal of Agriculture Sciences, ISSN: 0975-3710 \& E-ISSN: 0975-9107, Volume 4, Issue 5, pp-230-232.

Copyright: Copyright@2012 More B.M. This is an open-access article distributed under the terms of the Creative Commons Attribution License, which permits unrestricted use, distribution, and reproduction in any medium, provided the original author and source are credited.

\section{Introduction}

All plants growth is connected with light. In trees, the trunk brings the tree up into the light filled atmosphere. But as a rule, a tree trunk grows straight up; it is not directed towards the sun as a source of light, which is in fact a move daily across the sky. This growth straight upward is known as negative geotropism. Since the trunk grows directly away from the center of earth the blossoms of numerous wild flowers, in contrast, follow the path of the sun during the day; they exemplify positive phototropism- growing toward the light source [1].

The trees genetic propensity is to capture as much light as possible, which maximize its ability to do photosynthesis, grow and produce fertile offspring, which guarantee the survival of species. Since every tree has this propensity and growth creates shade that brings about death, each tree competes with its neighbor to maximize light uptake [2]

The trees are nearly symmetrical in shapes but sometimes they are asymmetrical. You might even call them misshapen [1]. Some of the twigs of some trees are BENDING DOWNWARDS [BD] in a straight line. This bending of twigs will have some specific reason. In this publication, I propose to outline the body of research that suggests a strong evolutionary connection between the two phenomena, bending of twigs of tree and presence of underground water stream.

\section{Growth of Trees}

The buds elongate the branches and widen the crown (Branches and leaves).

The particular shape of the crown and the size of the trunk relative to the crown in an individual tree express a different asset of the tree $[1,3]$. The neem tree in Fig. 1 gives USUAL GROWTH [UG] of tree and the crown nea.rly symmetrical in shape. The branches are growing towards the light. The crowns of some trees are markedly asymmetrical. You might even call them misshapen or UNUSUAL GROWTH [UUG] of some branches. Sometimes the twigs of these branches are BD direction as shown in Fig. 2. 


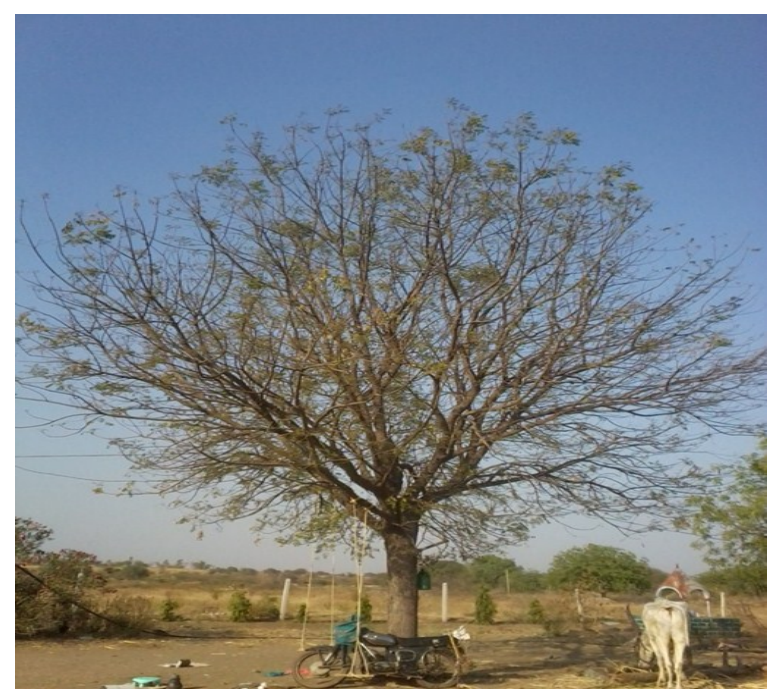

Fig. 1- Shows usual growth of Neem tree with branches nearly radial from the trunk

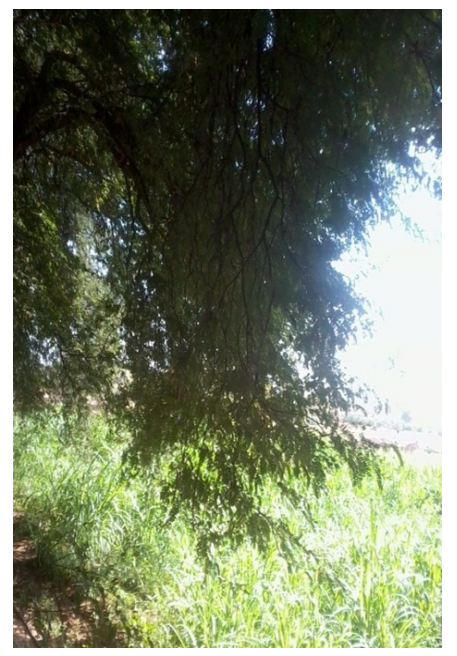

Fig. 2a-

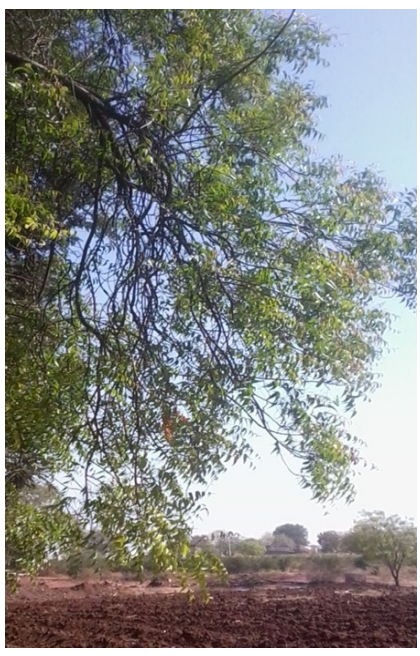

Fig. 2b-
Fig. 2- Unusual growth of trees with twigs bending downwards a) Tamarind and b) Neem

\section{New Technique of Searching of Underground water stream}

Generally there are many methods, vary widely; most dowsers (also called diviners or water witches) probably still use the traditional forked stick, which may came from variety of trees. Other dowsers may use keys, wire, coat hangers, pliers, wire rods and pendulum etc [4]. Locating underground water stream is both an art and by chance [5]. For drilling of bore well, no detail maps of ground water presence and no guarantee. Therefore it is quite confusing to take a decision about whether to drill or where to drill [6].

The technique by using UUG of trees is useful for finding place for drilling bore wells. The tree which has twigs BD gives ground surface direction of UWS. This will indicate one UWS stream surely. If we want two streams, we have to observe the same tree or other nearby trees, which are having the bending of twigs with different directions. Take a cross point of these two streams direction this will give you two streams may be at different depths. The cross point for drilling of bore well is calculated as shown in Fig. 3.

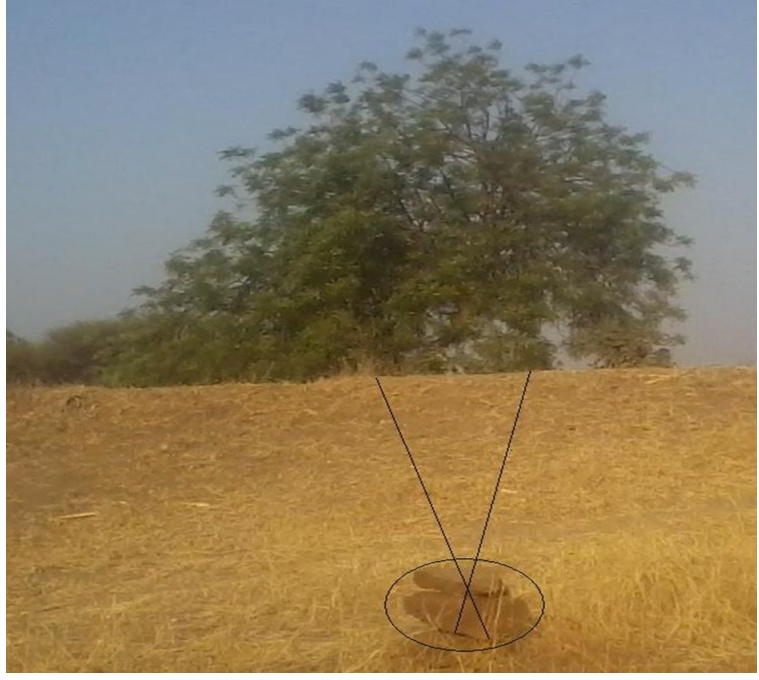

Fig. 3- A circle shows point for drilling bore well

\section{Case study}

This new technique was applied to Shri Raosaheb More's land, located at Tulshi, Tal Madha, Dist Solapur. The bore well point was fixed by considering UUG of two different trees and taking a cross point of these two. The bore was drilled on 13/03/2012 and more than 200 liter per minute water source was available during the drilling of bore well. The Fig. 4 shows the photograph of out coming water source at the time of drilling.

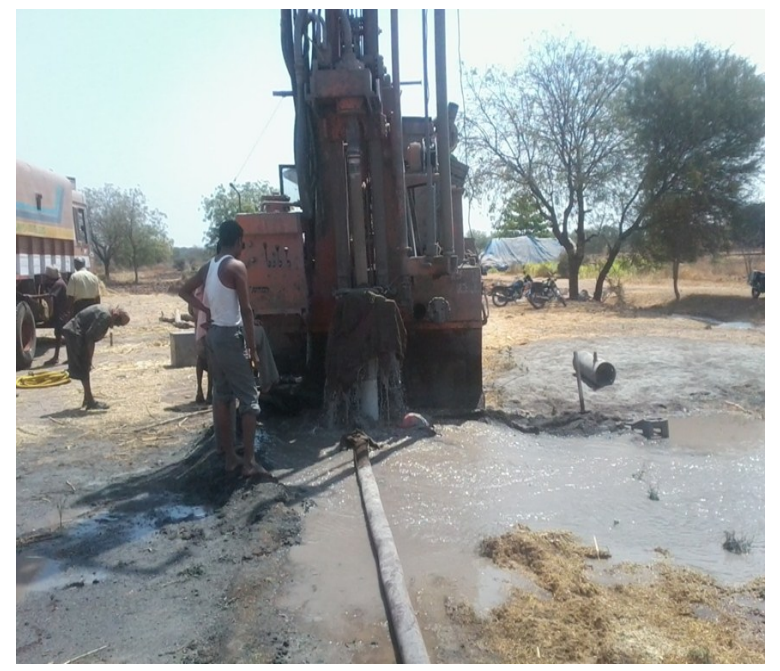

Fig. 4- Water coming out of bore well at the time of drilling

\section{Results and Discussion}

The trees of Neem and Tamarind shows UUG, as some of twigs are $\mathrm{BD}$ in a straight line. It means that in addition to gravitational force, there is additional downward force acting on these twigs. This additional force is might be due to presence of UNDERGROUND WATER STREAM [UWS]. There is abundant evidence from the trees on the banks of the river or nearby the wells, to support that the bending of twigs is due to presence of underground water, where we can see that twigs are bending downwards. Because of this continuous acting additional force, the twigs are stretched in downward direction. The comparison of length of twigs from UG and that of from UUG i.e., bending twigs, 
shows that the length of bending twigs is larger than that of UG. But, the diameter of sub branches of twigs which are bending is smaller. This is because of continuous additional force acting in downward direction. The bending of twigs is along a straight line. If a plane is drawn parallel to the direction of bending, it gives the direction of UWS on ground surface. We have to drill a bore well at this point on the ground surface.

\section{Conclusion}

In conclusion, the bending of some of twigs of some trees indicates presence of underground water stream. The bore well drilled under study might well support the fact. As the stream will not be always in straight line, the cross point of two or three streams sometime give less guarantee. The depth of stream varies from stream to stream, many times depth is up to 100 feet but sometimes it is up to 600 feet.

\section{Acknowledgement}

Author is very thankful to Prof. Snehal Mane, the Secretary of Brahmdevdada Mane Institute of Technology, Belati, Solapur and Dr. L.P. Deshmukh, Professor in Physics, Solapur University, Solapur, for their support and encouragement. I also thanks all my colleagues, who are involved in discussion of the idea of this article.

\section{References}

[1] http://natureinstitute.org/pub/ic/ic14/trees.html.

[2] Darwin C. (1979) The Origin of Species, Middlesex, Penguin Classics, Chapter III, 116.

[3] http:// texastreeid.tamu.edu/content/howTreeGrow/.

[4] http://ga.water.usgs.gov/edu/dowsing.html.

[5] http://dew.drop.com/purewateringroundstreams.html.

[6] Daneil S., Rex B. and Marios S. (2006) Kansas Geological Survey, Public information circular no. 23. 